\title{
Potential screening and early diagnosis method for cancer: Tongue diagnosis
}

\author{
SHUWEN HAN ${ }^{1}$, XI YANG $^{2}$, QUAN QI ${ }^{1}$, YUEFEN PAN $^{1}$, YONGCHAO CHEN $^{1}$, \\ JUNJUN SHEN $^{1}$, HAIHONG LIAO ${ }^{1}$ and ZHAONING JI ${ }^{3}$ \\ ${ }^{1}$ Department of Medical Oncology, Huzhou Central Hospital, Huzhou, Zhejiang 313000; \\ ${ }^{2}$ Department of Oncology, Wannan Medical College, Wuhu, Anhui 241000; ${ }^{3}$ The Cancer Center, \\ Yijishan Hospital of Wannan Medical College, Wuhu, Anhui 241001, P.R. China
}

Received February 9, 2016; Accepted March 4, 2016

DOI: $10.3892 /$ ijo.2016.3466

\begin{abstract}
Tongue diagnosis, as a unique method of traditional Chinese medicine (TCM), was used to discriminate physiological functions and pathological conditions by observing the changes of the tongue and tongue coating. The aims of the present study were to explore a potential screening and early diagnosis method of cancer through evaluating the differences of the images of tongue and tongue coating and the microbiome on the tongue coating. The DS01-B tongue diagnostic information acquisition system was used to photograph and analyze the tongue and tongue coating. The next-generation sequencing technology was used to determine the V2-V4 hypervariable regions of $16 \mathrm{~S}$ rDNA to investigate the microbiome on the tongue coating. Bioinformatics and statistical methods were used to analyze the microbial community structure and diversity. Comparing with the healthy people, the number of mirror-like tongue, thick tongue coating and the moisture of tongue were increased in cancers. The dominant color of the tongue in the healthy people was reddish while it was purple in the cancers. The relative abundance of Neisseria, Haemophilus, Fusobacterium and Porphyromonas in the healthy people were higher than that in the cancers. We also found 6 kinds of special microorganisms at species level in cancers. The study suggested that tongue diagnosis may provide potential screening and early diagnosis method for cancer.
\end{abstract}

\section{Introduction}

Traditional Chinese medicine (TCM) originated in ancient China and rooted in the ancient philosophy of naive materialism and natural dialectics. TCM, as a complete set of the theoretical system, is completely different from modern

Correspondence to: Dr Zhaoning Ji, The Cancer Center, Yijishan Hospital of Wannan Medical College, No. 2 Zheshan West Road, Wuhu, Anhui 241001, P.R. China

E-mail: 924441173@qq.com

Key words: cancer, tongue, tongue coating, microbiome, tongue diagnosis medicine (1). It is to explore the cause, nature, position and pathogenesis of the disease and maintain the health of human body basing on the guiding principles of the holistic concept and syndrome differentiation $(2,3)$ and the theory of Yin and Yang (the two opposing principles in nature) and the five elements (gold, wood, water, fire and soil). Four methods of diagnosis including observation diagnosis, auscultation, interrogation and feeling the pulse and numerous effective therapeutics including Chinese herbs (4), acupuncture (5), moxibustion, massage, cupping therapy, Tai chi, qigong (6) and so on, constitute a complete diagnosis and treatment system of TCM.

TCM is the practice of the Chinese people for thousands of years, many ideas and experience are worth further study (7-9). Perhaps the best example is that Tu Youyou gained inspiration from TCM to find artemisinin and won the Nobel Prize in Physiology or Medicine in 2015 (10). Tongue diagnosis is the vital component of observation diagnosis of TCM to examine the physiological and pathological changes of the human body. Tongue diagnosis, as a concept for the preventive treatment of diseases, originates from $\mathrm{Su}$ Wen (plain questions) (11). It is a simple, non-invasive and valuable diagnostic procedure that has been repeatedly verified by clinical practitioners of TCM for at least 2,000 years $(12,13)$. However, the TCM and modern medicine was established on experience and experiment, respectively. Tongue diagnosis was not widely used in clinical practice of modern medicine because doctor's experience affects the objectivity and reproducibility of tongue diagnosis (14). With the development of modern computer and molecular biology technology, the quantitative research of tongue diagnosis should be brought into schedule (15).

In the clinic, the commonly used diagnostic methods of cancer are: imageological diagnosis, ultrasonic diagnosis, endoscopic diagnosis, serological indicator, pathological diagnosis and so on. Although huge progress has been made in cancer diagnostics, we cannot help but admit that a high number of cases of cancers have already metastasized when diagnosed. Therefore it is necessary to find new methods as supplement to cancer screening and early diagnosis. We consider that the tongue diagnosis, based on TCM, shows promise and can provide some help. 
Table I. Characteristics of study participants.

\begin{tabular}{|c|c|c|c|c|c|c|c|}
\hline & $\begin{array}{l}\text { Control } \\
\text { group }\end{array}$ & $\begin{array}{l}\text { Colorectal } \\
\text { cancer }\end{array}$ & $\begin{array}{l}\text { Lung } \\
\text { cancer }\end{array}$ & $\begin{array}{l}\text { Gastric } \\
\text { cancer }\end{array}$ & Cancers & $\chi^{2} /$ F-value & P-value \\
\hline Cases, $\mathrm{n}$ & 100 & 90 & 96 & 100 & 286 & - & NA \\
\hline Males, n & 51 & 43 & 55 & 49 & 147 & 2.04 & 0.728 \\
\hline Age, years & $53.57 \pm 8.32$ & $55.45 \pm 11.55$ & $55.14 \pm 9.80$ & $56.20 \pm 10.24$ & $55.58 \pm 10.53$ & 1.175 & 0.319 \\
\hline BMI, $\mathrm{kg} / \mathrm{m}^{2}$ & $23.60 \pm 2.57$ & $24.04 \pm 3.12$ & $23.42 \pm 3.19$ & $22.92 \pm 3.33$ & $23.44 \pm 3.24$ & 2.155 & 0.093 \\
\hline Current smoker, $\mathrm{n}$ & 7 & 6 & 11 & 6 & 23 & 2.478 & 0.649 \\
\hline Known diabetes, $\mathrm{n}$ & 3 & 7 & 3 & 2 & 12 & 4.846 & 0.304 \\
\hline Known hypertension, $\mathrm{n}$ & 3 & 2 & 3 & 2 & 7 & 0.384 & 0.984 \\
\hline I stage & - & 16 & 16 & 28 & 60 & 5.202 & 0.816 \\
\hline II stage & - & 38 & 44 & 41 & 123 & & \\
\hline III stage & - & 28 & 29 & 24 & 81 & & \\
\hline IV stage & - & 8 & 7 & 7 & 22 & & \\
\hline
\end{tabular}

NA, not applicable due to selection criteria. The characteristic of the healthy people and patients with cancers was described. The clinical stages were according to the AJCC (American Joint Committee On Cancer) cancer staging guidelines. The categorical data analysis and measurement data were performed with the $\chi^{2}$ test and analysis of variance, respectively. The results of P-value showed the difference among the groups had no statistical significance $(\mathrm{P}>0.05)$.

In the present study, the technology of computer-aided medical image processing and analysis and microbic genomics were used to study the images of tongue and tongue coating and the microorganisms on the tongue coating, respectively. The evidence was intended to show that tongue diagnosis is a potential method for cancer screening and early diagnosis by comparing the differences between the healthy people and the patients with cancers.

\section{Materials and methods}

Patients with cancer and healthy controls. A total of 286 patients with cancers were recruited from the Cancer Center of Yijishan Hospital of Wannan Medical College and the Department of Medical Oncology of Huzhou Center Hospital from September 2013 to September 2015. There are 90 patients with colorectal cancer, 96 patients with lung cancer and 100 patients with gastric cancer. All the cancers were confirmed by pathological diagnosis and the clinical stages were according to the AJCC (American Joint Committee On Cancer) cancer staging guidelines. One hundred healthy controls were recruited from the Health Examination Center of Huzhou Center Hospital. The healthy controls had no digestive diseases, no respiratory diseases, no oral disease, no malignant tumor and cancer related symptoms in the last two years. All of the subjects signed informed consent under the guideline approved by the Ethics Committee of Huzhou Central Hospital. The characteristics of the study participants are shown in Table I.

The images of tongue and tongue coating analysis. The participants were required to rinse their mouth before the examination was performed. The DS01-B tongue diagnostic information acquisition system (Daosh Co., Shanghai, China) included a camera and a computer. The image analysis and processing software, by DAOSH company, was used to photograph and analyze the images of the tongue and tongue coating of all the participants. The analysis of the contents of tongue coating included the presence or absence and the thickness of the tongue coating and the tongue characteristics such as the tongue color, moisture, greasiness, indentation, pricking, fissure and ecchymosis.

The samples collection. The samples were collection in the morning prior to breakfast. All participants were required to rinse their mouth before sampling. The front and middle section of the tongue coating was scraped by a sterile spoon and put into the test tube with saline. Repeated 3 times and centrifuged $(2,000 \mathrm{r} / \mathrm{min}) 5 \mathrm{~min}$. The sediment was stored in the ultra-low temperature freezer until used.

MiSeq sequencing. The next-generation sequencing technology was used to determine the V2-V4 hypervariable region of $16 \mathrm{~S}$ rDNA to investigate the microbiome on the tongue coating. The integrity of genome DNA from each tongue coating sample was evaluated using a TRIzol ${ }^{\circledR}$ reagent (Invitrogen, Carlsbad, CA, USA) according to the manufacturer's recommended protocol. PCR amplification was conducted with the minimum cycle number. The sequences of the V2-V4 hypervariable region of $16 \mathrm{~S}$ rDNA were as follows: V2-V4 (338Fe806R): 338 F, ACTCCTACGGGAGGCAGCA; 806R, GGACTACHVGGGTWTCTAAT. The obtained PCR products were run on $2 \%$ agarose gel and purified after size selection. Repeated three times for each sample and quantified using QuantiFluor ${ }^{\mathrm{TM}}$ ST system. The MiSeq library was constructed as follows: PCR products were ligated with $\mathrm{Y}$ adapter. Magnetic nanoparticles were used to take out the selfligated Y adapters. PCR amplification was conducted for library enrichment and treated with $\mathrm{NaOH}$ solution. The single-stranded DNA sequences were acquired and sequenced 

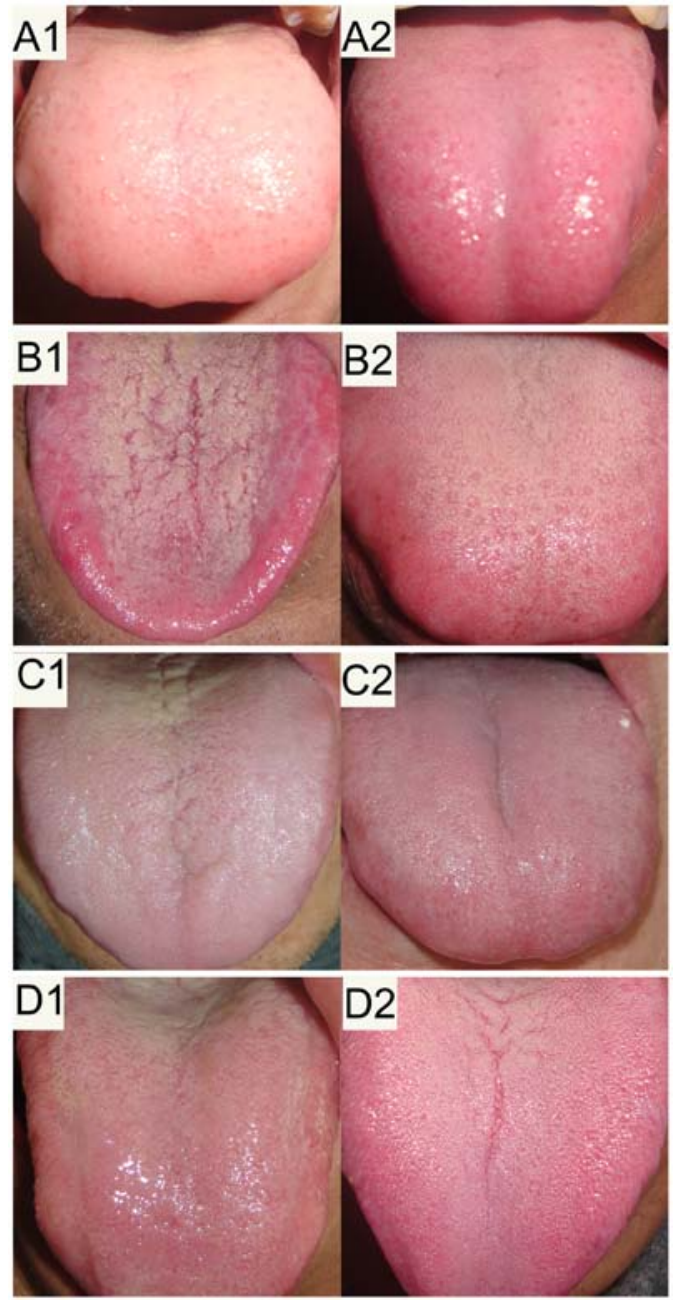

Figure 1. Tongue images.

on the MiSeq platform (Majorbio Bio-pharm Biotechnology Co., Ltd. Shanghai, China) according to the standard protocols (16).

\section{Data analysis}

Sequencing data bioinformatics analysis. The sequencing data were filtered and trimmed and then used for clustering OTU (operational taxonomic units) and Taxonomic analysis. The seqcln software was employed to examine the adapter and 3 ' end sequence and the mothur software (http://www.mothur. org/wiki/Main_Page) was used for the read filtering. The raw data were eliminated as follows: the reads with more than 2 mismatch bases in the forward primer; the low quality bases from the 3 ' end; the ambiguous bases, or short ( $<200 \mathrm{bp}$ ) reads, or reads with average accuracy less than Q25. The mothur software was employed to analyze OTU cluster at $97 \%$ identity to estimate the number of species or genus in each sample. The database of SILVA and the mothur software were used to analyze the clean reads and to acquire the taxonomy information. The R software was used to make the Venn Chart.

Statistical analysis. The SPSS software (v.16.0; SPSS, Inc., Chicago, IL, USA) was used for statistical analyses. The data were expressed as the mean \pm standard deviation (SD). Chi-square test test or Fisher's exact test was calculated for
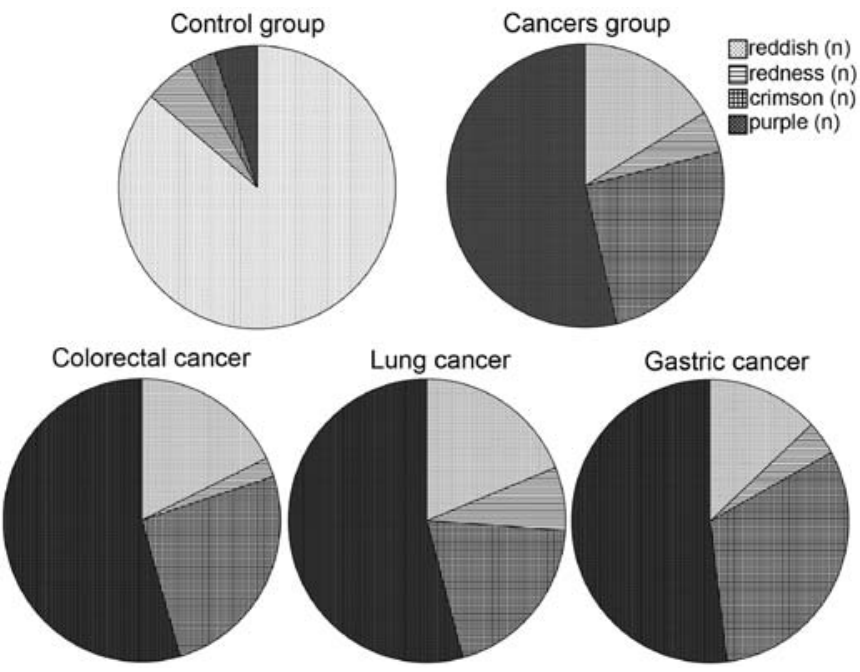

Figure 2. Tongue color.

categorical variables. The t-test and q-test were used to compare the means. Statistical significance was set at $\mathrm{P}<0.05$.

\section{Results}

The images characteristic of the tongue coating in the cancers and healthy people. In the TCM, observation of tongue coating is one of the important methods for diagnosing diseases. Tongue coating defined as a thin and wet layer on the surface of tongue consists of epithelium, saliva, microorganism and food debris. There is no unified standard to assess the thickness of tongue coating. The tongue diagnostic information acquisition system was performed to analyze the thickness of tongue coating basing on the Bayes probability formula. It was considered to be absence or less tongue coating if the value was $<110$. The scope of the value of thin tongue-coating and thick tongue-coating was 110-300 and 300-800, respectively. Mirror-like tongue was defined that the tongue coating was very smooth and the thickness of the tongue coating was negligible. As shown in Fig. 1, the $\mathrm{A} 1$ and $\mathrm{A} 2$ images were from the healthy people and the others were from cancers. The tongue coating in A1, A2, $\mathrm{B} 1, \mathrm{C} 1$ and D1 images were thin and the others were thick. Comparing with 100 cases of healthy people and 286 cases of cancers, the number of mirror-like tongue in the patients with cancers was greater than that in the healthy people (Table II; P<0.001). However, comparing the thickness of tongue coating except mirror-like tongue, the thickness of tongue coating in the patients with cancers was thicker than that in the healthy people (Table III; $\mathrm{P}<0.001$ ).

The images characteristic of the tongue in the cancers and healthy people. The tongue diagnostic information acquisition system was used to describe the tongue color, moisture, greasiness, indentation, pricking, fissure and ecchymosis in the cancers and healthy people. The color of tongue was classified into four categories including reddish, redness, crimson and purple. Through comparative analysis of the color of tongue in 100 healthy people and 286 cancer patients, the tongue color in healthy people was mainly reddish, but the tongue color 
Table II. The comparison of the cases number of the tongue coating.

\begin{tabular}{lccccc}
\hline Category & Control group & Colorectal cancer & Lung cancer & Gastric cancer & Cancers \\
\hline Mirror-like tongue & 13 & 45 & 64 & 74 & 183 \\
Tongue coating & 87 & 45 & 32 & 26 & 103 \\
$\chi^{2}$ value & - & 30.577 & 59.142 & 75.699 & 77.063 \\
P-value & - & $<0.001$ & $<0.001$ & $<0.001$ & $>0.001$ \\
\hline
\end{tabular}

Tongue coating, as a TCM term, is a thin and wet layer on the surface of tongue. It is composed of epithelium, saliva, microorganism, food debris and so on. The mirror-like tongue is the tongue coating on a person that can not be detected. The tongue diagnostic information acquisition system was used to describe the presence or absence of the tongue coating objectively. The table shows that the number of mirror-like tongue in the patients with cancers was more than that in the healthy people $(\mathrm{P}<0.001)$.

Table III. The comparison of the thickness of the tongue coating.

\begin{tabular}{lccccc}
\hline Category & Control group & Colorectal cancer & Lung cancer & Gastric cancer & Cancers \\
\hline Cases $(n)$ & 87 & 45 & 32 & 26 & 103 \\
Thickness & $204.74 \pm 44.81$ & $592.08 \pm 137.93$ & $574.59 \pm 168.39$ & $508.91 \pm 155.74$ & $565.65 \pm 154.67$ \\
t-value & - & 23.94 & 18.87 & 16.25 & 21.03 \\
P-value & - & $<0.001$ & $<0.001$ & $<0.001$ & $<0.001$ \\
\hline
\end{tabular}

The tongue diagnostic information acquisition system was used to describe the thickness of the tongue coating. There is no unified standard to assess the thickness of tongue coating. The tongue diagnostic information acquisition system was performed to analyze the thickness of tongue coating basing on the Bayes probability formula. It was considered to be absence or less tongue coating if the value was $<110$. The scope of the value of thin tongue-coating and thick tongue-coating was 110-300 and 300-800, respectively. The table shows that the thickness of tongue coating in the patients with cancers was more than that in the healthy people $(\mathrm{P}<0.001)$.

Table IV. The comparison of the tongue color.

\begin{tabular}{|c|c|c|c|c|c|}
\hline Category & Control group & Colorectal cancer & Lung cancer & Gastric cancer & Cancers \\
\hline Case number (n) & 100 & 90 & 96 & 100 & 286 \\
\hline Reddish (n) & 86 & 16 & 18 & 13 & 47 \\
\hline Redness (n) & 6 & 2 & 7 & 4 & 13 \\
\hline Crimson (n) & 3 & 23 & 19 & 31 & 73 \\
\hline Purple (n) & 5 & 49 & 52 & 52 & 153 \\
\hline$\chi^{2}$ value & - & 101.00 & 94.89 & 116.00 & 166.1 \\
\hline P-value & - & $<0.001$ & $<0.001$ & $<0.001$ & $<0.001$ \\
\hline
\end{tabular}

The tongue diagnostic information acquisition system was used to describe the tongue color. The color of tongue was classified into four categories including reddish, redness, crimson and purple. One hundred healthy people and 286 cancer patients were recruited in the present study. The rate of the reddish tongue color in the healthy people was higher than that in the cancer patients, while the rate of purple color in the cancer patients was higher.

in cancers was mainly purple (Fig. 2), and the results were statistically significant (Table IV; $\mathrm{P}<0.001$ ).

The moisture, indentation, pricking, fissure and ecchymosis of tongue in the healthy people was higher than that in cancers and the difference had statistical significance (Fig. 3 and Table IV; $\mathrm{P}<0.05)$. The large discrete tendency of data distribution indicated that the other factors may affect the indentation, pricking and ecchymosis of the tongue. It may be limited by the tongue diagnostic information acquisition system, the ceiling of the fissure numbers of tongue was 2000 , but the fissure numbers of tongue in the majority of cancer patients was over 2000. Actually, the difference of the fissure numbers of tongue between the healthy people and cancer patients was clearly noticeable.

The tongue coating microbiome in the cancers and healthy people. The microbiome on the tongue coating was investigated by sequencing the V2-V4 hypervariable region of $16 \mathrm{~S}$ rDNA. 

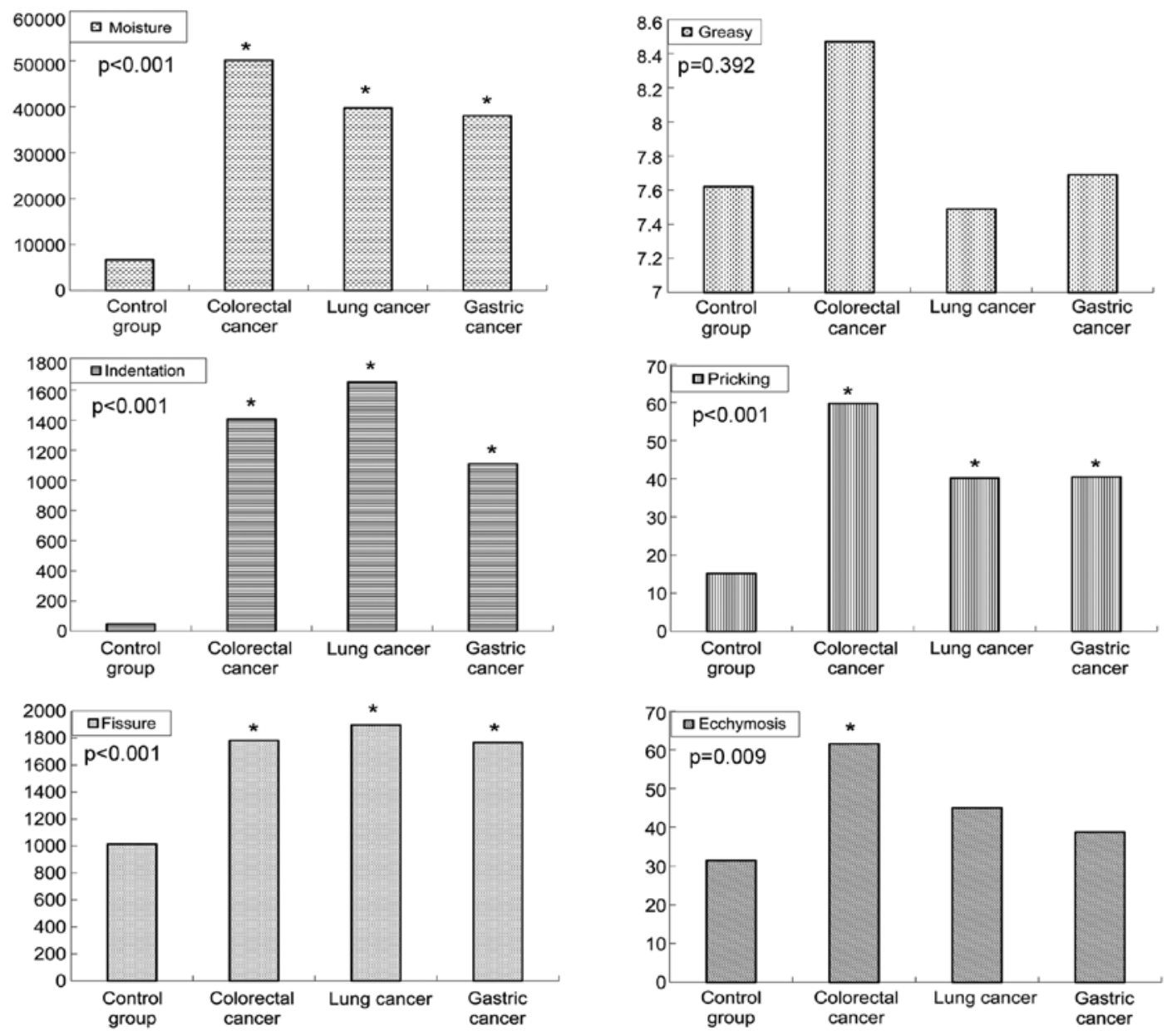

Figue 3. Images characteristic of the tongues in healthy people and cancer patients.

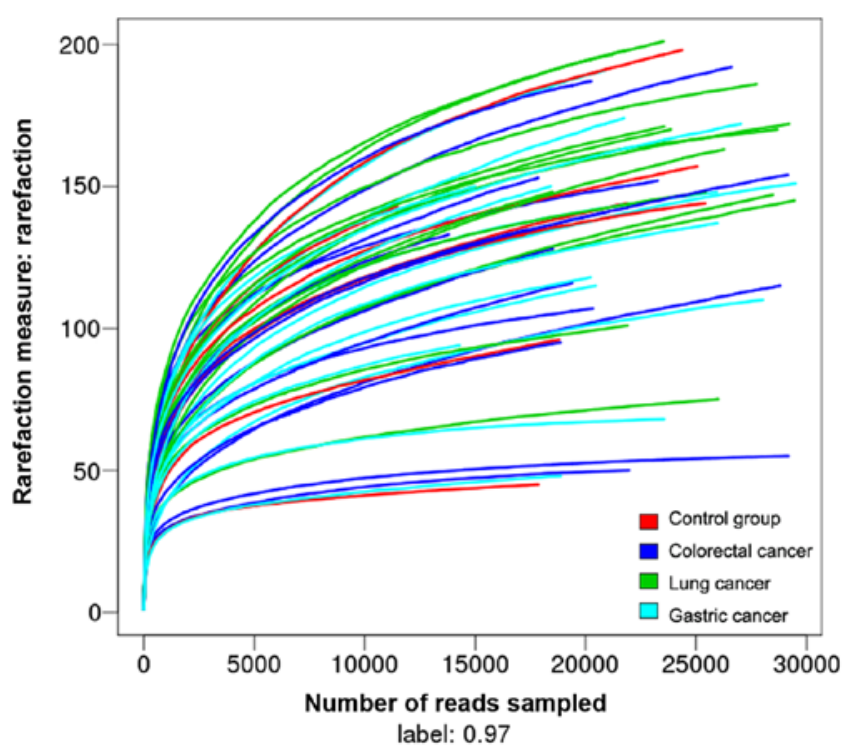

Figure 4. Rarefaction OTU of the tongue coating samples.

The sequencing data were filtered and trimmed and mothur software was used to analyze OTU cluster at $97 \%$ identity and the taxonomy information. The OTU (operational taxonomic units) is the artificial classification unit which is utilized in the phylogenetics and population genetics studies (17). Fig. 4

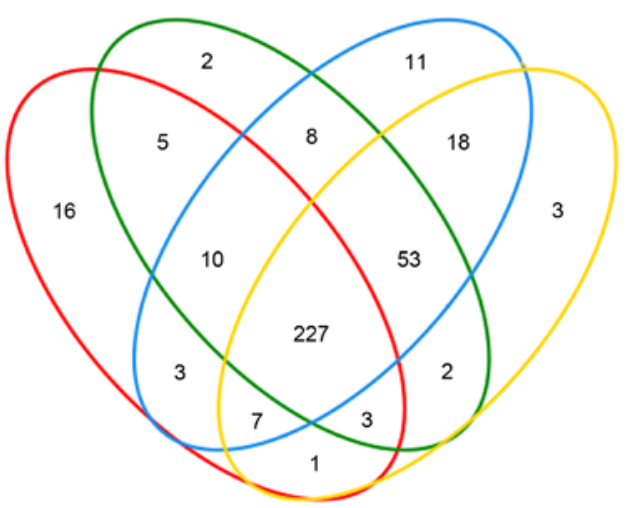

Unique objects: $A l l=369$

Control group: $\mathrm{S} 1=272$

Colorectal cancer: $\mathbf{S} 2=310$

Lung cancer: $\mathbf{S 3}=\mathbf{3 3 7}$

Gastric cancer: $\quad$ S4=314

Figure 5. Venn Chart basing on the OTUs.

showed the rarefaction OUT of 50 cases of tongue coating samples.

The Venn Chart was drawn to visualize the number of OTUs which are exclusive or shared by multiple samples. As shown in Fig. 5, the unique objects were 369, the control group was 272 , the colorectal cancer were 310 , the lung cancer 337 

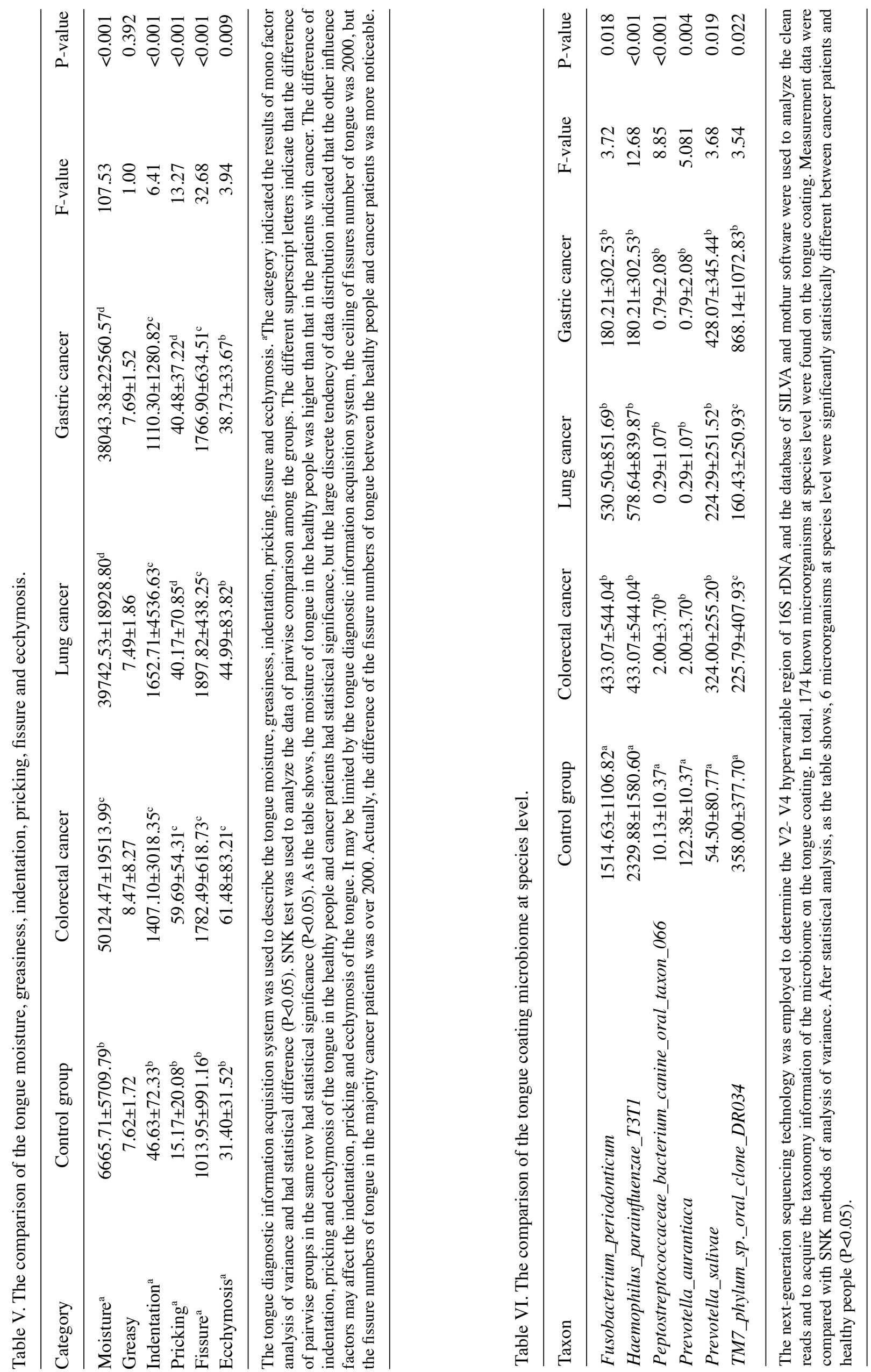


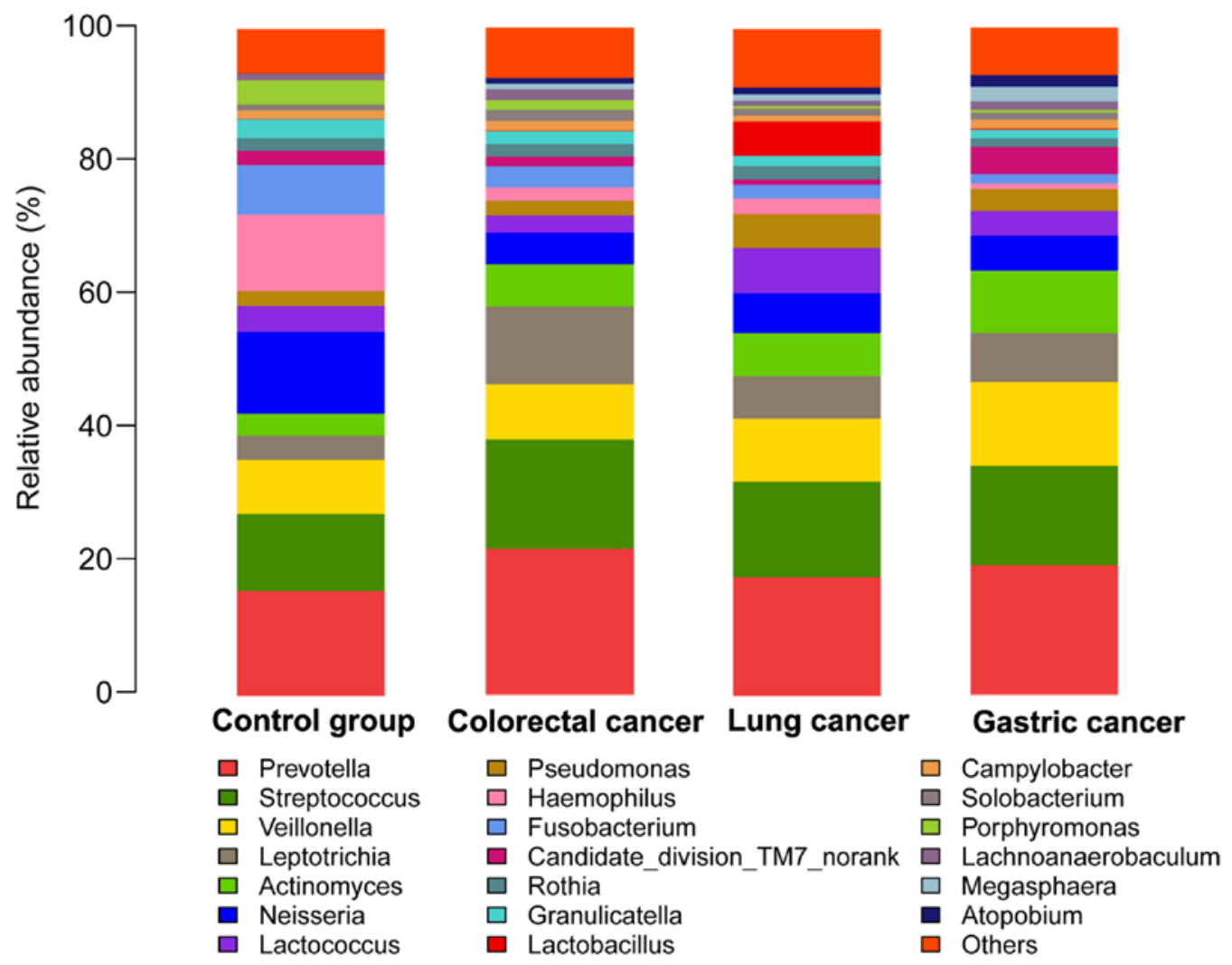

Figure 6. Microorganism community structure on the tongue coating.

and the gastric cancer were 314. This Venn diagram indicated the universalities and individualities of the microorganism on the tongue coating in the control group and several types of cancers.

The bioinformatics technology was employed to analyze the microbial community structure and diversity on the tongue coating. Fig. 6 describes the relative abundance of 22 microorganisms at the genus level. The relative abundance of Neisseria, Haemophilus, Fusobacterium and Porphyromonas in the control group was obviously higher than that in the cancer groups. Additionally, the 174 known microorganisms at species level were analyzed further, and there was statistical difference between the healthy people and cancer patients in the six species of microorganisms, Fusobacterium periodonticum, Haemophilus_parainfluenzae_T3T1, Peptostreptococcaceae_bacterium_canine_oral_taxon_066, Prevotella_aurantiaca, Prevotella_salivae and TM7 phylum_sp._oral_clone_DR034 (Table VI; P<0.05).

\section{Discussion}

The tongue is a very special organ. It is not only the part of digestive system, but also associated with respiratory system. It is in direct contact with the external environment when the mouth is opened, while it is not when the mouth is closed. In addition, the saliva, microorganism and food particles remain on the tongue coating long-term. It was finally decided that the images of tongue and tongue coating vary with different individuals. As is known, multiple factors such as dietary habits (18), life styles (19) and micro-ecological environment (20) are involved in the formation of cancer. Similarly, these factors are reflected on the tongue. Above all, these aspects supported the tongue diagnosis as a potential screening and early diagnosis method to cancer.

The tongue diagnostic information acquisition system was previously used to analyze the images of tongue and tongue coating $(13,21)$. In the present study, we analyzed the images characteristic of the tongue and tongue coating in the colorectal, lung and gastric cancers and healthy people. The characteristics of the tongue and tongue coating in the cancers indicated that the tongue diagnosis may provide potential screening and early diagnosis method to cancer. It is undeniable that further investigation of larger sample sizes and more precise data are required.

In order to explore the underlying reason of the specific images, the microbiome on the tongue coating was detected and analyzed. The microbial community structure and diversity by analyzing the OTUs and known microorganisms at genus and species levels. The six specific microorganisms at species level in the cancers may point out further research directions of the tongue diagnosis. More research is needed to confirm the relationship between the specific microorganisms and images characteristic of the tongue and tongue coating. The biology of the microbiome is still poorly known (22), despite the Human Mcrobiome Poject that was put forward in 2007 (23). The development of microbiology and the bioinformatics technology and the falling price of gene sequencing, more species and more detailed biological characteristics of microorganisms can be discovered. The more valuable clues of the microorganisms on the tongue coating of cancers may be confirmed as the potential microbiological indicators of screening and early diagnosis to cancer in the future. 


\section{Acknowledgements}

We thank patients and volunteers for their contributions in images and samples collection.

\section{References}

1. Han S, Chen Y, Hu J and Ji Z: Tongue images and tongue coating microbiome in patients with colorectal cancer. Microb Pathog 77: 1-6, 2014

2. Zhang P: Advantages, disadvantages, and trend of integrative medicine in the treatment of heart failure. Cell Biochem Biophys 72: 363-366, 2014

3. Fu J, Pang J, Zhao $X$ and Han J: The quantitative ideas and methods in assessment of four properties of chinese medicinal herbs. Cell Biochem Biophys 71: 1307-1312, 2014.

4. Ko RJ: A U.S. perspective on the adverse reactions from traditional Chinese medicines. J Chin Med Assoc 67: 109-116, 2004.

5. Yin LM, Wang Y, Fan L, Xu YD, Wang WQ, Liu YY, Feng JT, Hu CP, Wang PY, Zhang TF, et al: Efficacy of acupuncture for chronic asthma: Study protocol for a randomized controlled trial. Trials 16: 424, 2015

6. Birdee GS, Wayne PM, Davis RB, Phillips RS and Yeh GY: T'ai chi and qigong for health: Patterns of use in the United States. J Altern Complement Med 15: 969-973, 2009.

7. Li YZ, Li GZ, Gao JY, Zhang ZF, Fan QC, Xu JT, Bai GE, Chen $\mathrm{KX}$, Shi HZ, Sun S, et al: Syndrome differentiation analysis on Mars500 data of traditional Chinese medicine. Sci World J 2015 $125736,2015$.

8. Li X, Xiao QQ, Ze K, Li S, Wang YF, Zhou M, Yang QT, Li FL and Li B: External application of traditional Chinese medicine for venous ulcers: A systematic review and meta-analysis. Evid Based Complement Alternat Med 2015: 831474, 2015.

9. Liu J, Wang S, Zhang Y, Fan HT and Lin HS: Traditional Chinese medicine and cancer: History, present situation, and development. Thorac Cancer 6: 561-569, 2015.

10. Tu Y: The discovery of artemisinin (qinghaosu) and gifts from Chinese medicine. Nat Med 17: 1217-1220, 2011

11. Cui Y, Liao S, Wang H: ROC-Boosting: A feature selection method for health identification using tongue image. Comput Math Methods Med 2015: 362806, 2015.
12. Jiang B, Liang X, Chen Y, Ma T, Liu L, Li J, Jiang R, Chen T, Zhang $X$ and Li S: Integrating next-generation sequencing and traditional tongue diagnosis to determine tongue coating microbiome. Sci Rep 2: 936, 2012.

13. Lo LC, Cheng TL, Chen YJ, Natsagdorj S and Chiang JY: TCM tongue diagnosis index of early-stage breast cancer. Complement Ther Med 23: 705-713, 2015.

14. Kim J, Han G, Ko SJ, Nam DH, Park JW, Ryu B and Kim J: Tongue diagnosis system for quantitative assessment of tongue coating in patients with functional dyspepsia: A clinical trial. J Ethnopharmacol 155: 709-713, 2014.

15. Gareus I, Rampp T, Tan L, Lüdtke R, Niggemeier C, Lauche R, Klose $\mathrm{P}$ and Dobos G: Introducing a computer-assisted, digital tongue-imaging device for standardization of traditional tongue diagnosis. Forsch Komplement Med 21: 190-196, 2014.

16. Yu H, Wang Q, Wang Z, Sahinkaya E, Li Y, Ma J and Wu Z: Start-up of an anaerobic dynamic membrane digester for waste activated sludge digestion: Temporal variations in microbial communities. PLoS One 9: e93710, 2014.

17. Lynch MD, Bartram AK and Neufeld JD: Targeted recovery of novel phylogenetic diversity from next-generation sequence data. ISME J 6: 2067-2077, 2012.

18. Campagna M, Cocco P, Zucca M, Angelucci E, Gabbas A Latte GC, Uras A, Rais M, Sanna S and Ennas MG: Risk of lymphoma subtypes and dietary habits in a Mediterranean area. Cancer Epidemiol 39: 1093-1098, 2015.

19. Alvarez-Cubero MJ, Pascual-Geler M, Rivas A, MartinezGonzalez LJ, Saiz M, Lorente JA and Cozar JM: Lifestyle and dietary factors in relation to prostate cancer risk. Int J Food Sci Nutr 66: 805-810, 2015.

20. Vento $\mathrm{S}$ and Tanko MN: The bacterium that could cause cancer. Lancet Oncol 10: 528, 2009.

21. Yamamoto S, Ishikawa Y, Nakaguchi T, Ogawa-Ochiai K, Tsumura N, Kasahara Y, Namiki T and Miyake Y: Temporal changes in tongue color as criterion for tongue diagnosis in Kampo medicine. Forsch Komplement Med 19: 80-85, 2012.

22. Integrative HMP; Integrative HMP (iHMP) Research Network Consortium: The Integrative Human Microbiome Project: Dynamic analysis of microbiome-host omics profiles during periods of human health and disease. Cell Host Microbe 16: 276-289, 2014.

23. Turnbaugh PJ, Ley RE, Hamady M, Fraser-Liggett CM, Knight R and Gordon JI: The human microbiome project. Nature 449: 804-810, 2007. 